\title{
Survival of in-hospital cardiac arrest in men and women in a large Swedish cohort
}

\author{
Angelika Qvick', Manar Radif', Caroline Brever', Jenny Olsson Myrvik', Karin Schenk Gustafsson² \\ and Therese Djärv ${ }^{1,3^{*}}$
}

\begin{abstract}
Background: Cardiac arrest is more common in men than women and a few studies have shown inferior 30-day survival for men than for women. The difference might relate to patient characteristics, intra arrest factors or post arrest care.

Aim: To assess differences in 30-day survival between men and women after an in-hospital cardiac arrest (IHCA). Material and methods: All patients $\geq 18$ years suffering an IHCA at Karolinska University Hospital between 2007 and 2017 were included. Data regarding the IHCA, patient characteristics, Charlson co-morbidity index (CCI) and 30-day survival were obtained from electronic patient records. Differences in survival between men and women were assessed with adjusted logistic regression models and presented as Odds Ratios with 95\% Confidence Intervals (OR, 95\% Cl). Adjustments included age, CCl, place of cardiac arrest, first rhythm, ECG-surveillance and witnessed or not.

Results: In all, 1639 patients suffered an IHCA, of whom 650 (40\%) were women and 193 (30\%) of them survived to 30 days compared to $28 \%$ of the men. No differences were found in the studied patient characteristics, intra arrest factors or post-ROSC treatments. Men had similar survival as women (crude OR 0.93 95\% Cl 0.74-1.15 and adjusted OR 0.77 95\% Cl 0.58-1.03 respectively).

Conclusion: This cohort study illuminates an almost equal distribution in characteristics and treatment as well as outcome, 30-day survival after IHCA between men and women. However, our study confirms previous findings of disadvantageous prerequisites among women, but also indicates that preceeding vital signs differ which might indicate residual confounding.
\end{abstract}

Keywords: Gender, Sex, 30-day survival, IHCA

\section{Introduction}

The impact of sex on survival after cardiac arrest remains in the current situation somewhat unclear with conflicting results. Previous studies show that men are overrepresented in the cardiac arrest population, constituting approximately $60 \%$ of all cases but have about a $10 \%$ lower chance of 30-day survival than women [1-3]. This decreased survival for men has been shown despite unfavourable prehospital factors among out-of-hospital cardiac arrests (OHCA) such as higher age, fewer witnessed arrests and less likely to receive bystander-CPR

\footnotetext{
* Correspondence: therese.djarv@ki.se

${ }^{1}$ Function of Emergency Medicine, Karolinska University Hospital, Stockholm, Sweden

${ }^{3}$ Department of Medicine Solna, Karolinska Institutet, Stockholm, Sweden

Full list of author information is available at the end of the article
}

for women [3]. Also, women with in-hospital cardiac arrest (IHCA) have both higher age and prevalence of non-shockable first rhythm [2]. The phenomenon that these studies demonstrate has been described as the 'gender paradox', where women survive cardiac arrests to a greater extent despite disadvantageous prerequisites [4]. One explanatory factor proposed for this 'gender paradox', have been that women are older when getting coronary artery disease, the reason for this is still unsolved, a so-called cardiovascular protective 'oestrogen effect' has been proposed [3, 5]. Another explanatory factor was presented in an American study from 2014, where female gender was associated with longer CPR-duration in patients with an IHCA not achieving ROSC [6].

(c) The Author(s). 2018 Open Access This article is distributed under the terms of the Creative Commons Attribution 4.0 International License (http://creativecommons.org/licenses/by/4.0/), which permits unrestricted use, distribution, and reproduction in any medium, provided you give appropriate credit to the original author(s) and the source, provide a link to the Creative Commons license, and indicate if changes were made. The Creative Commons Public Domain Dedication waiver (http://creativecommons.org/publicdomain/zero/1.0/) applies to the data made available in this article, unless otherwise stated. 
Still, there are conflicting results and sex has not been shown as a prognostic factor for survival after an IHCA [7]. In summary, women appear to have better outcome than men regarding cardiac arrests and the causal factor of this phenomenon is yet unknown. Therefore, we conducted a cohort study with the aim of assessing differences in patient characteristics, intra arrest factors as well as post-ROSC treatments between men and women as well as the association with gender and 30-day survival after an IHCA.

\section{Method}

\section{Study design and settings}

This cohort study took place at Karolinska University Hospital (Karolinska) between 1st January 2007 and 31st December 2017. Karolinska, in Stockholm, home to approximately 2,000,000 people, is one of five large Stockholm hospitals. Karolinska has two sites $30 \mathrm{~km}$ apart, Solna and Huddinge. The Solna site hosts about 750 beds and is a level one trauma unit, has neuro and thoracic surgery units and provides 24/7 angiography for ST-elevation myocardial infarctions. The Huddinge site also hosts about 750 beds - and relatively fewer intensive care unit (ICU) beds. Karolinska as a whole has about 108,000 admissions yearly and 1.8 million patient visits. Karolinska follows Swedish guidelines for resuscitation based on European Resuscitation Councils guidelines.

\section{Ethics}

This study used a database and registry: all survivors are informed about their participation at 6 months after their IHCA and can at any time afterwards withdraw their inclusion in the Swedish Registry for Cardio-Pulmonary Resuscitation (SRCR). Since the start of the registry in 1990 only a handful of patients have withdrawn their participation. Non-survivors are included without informed consent. The Regional Ethical Review Board in Stockholm, Sweden approved the study, Dnr 2013/1959-31/4.

\section{Participants}

All cases of IHCA among adults, i.e. patients aged at least 18 years, occurring between January 1st 2007 and December 31th 2017 were eligible for inclusion in the study and were identified through the hospital's cardiac arrest report sheet which is developed for collecting variables for participation in the SRCR. The SRCR $[8,9]$ collects data according to Utstein template [10], and the SRCRs definition of IHCA was used in the current study, i.e. a hospitalised patient who is unresponsive with apnoea (or agonal, gasping respiration) where CPR and/or defibrillation have been initiated. No patients or location of the IHCA were excluded. In the case of multiple IHCAs, only the first event was included.

\section{Data collection and categorisation}

Patients were identified through the hospital's cardiac arrest report sheet, where data on the following variables were collected: sex, age (collected in years, categorised in 10-year intervals starting at 18-50, 51-60 and further on to $>81$ years), location of IHCA (general ward, intermediate care unit, intensive care unit (ICU), angio lab/operation theatre or other area including emergency department and radiology department), and first documented heart rhythm (VT/VF or PEA/asystole). Thereafter by entering the hospital's electronic patient record (Take Care version 14.2.9) information on co-morbidities was gathered based on ICD-10 codes available at least at admission to the hospital and assessed according to the Charlson co-morbidity index (CCI) $[11,12]$ and categorised into "None" if CCI was 0 points, "Low burden of co-morbidities" if the CCI was 1-2 points, "Moderate burden of co-morbidities" if the CCI was 3-5 points or "High burden of co-morbidities" if the CCI was at least 6 points.

For a subset of the population, i.e. IHCA occurring 2015-2017 information on two additional variables was available in the medical file and therefore collected, i.e. information on National Early Warning Score (NEWS) [13] as well as information of a decision to withhold or withdraw life-sustainable treatment post- ROSC. Information regarding NEWS was collected as close as possible and maximum $12 \mathrm{~h}$ before the IHCA and categorized into low (0-4 points), medium (5-6 points or 3points in one single parameter) or high (at least 7 points) if information was available on all 7 included parameters.

Information on the outcome, i.e. 30-day survival (yes or no) was retrieved through the electronic patient record which is linked to the Swedish total population registry and automatically updated within a maximum delay of three days, which enables a complete follow-up [14].

\section{Statistical analyses}

Characteristics of women and men were compared using the two-sided Chi [2] test and a $p$-value of $\leq 0.05$ was interpreted as statistically significant. Missing data were not excluded or imputed, yet instead kept as an own category per variable. In order to assess the association between gender and 30-day survival, a logistic regression model was used and results were expressed as odds ratios (OR) with $95 \%$ confidence intervals $(95 \% \mathrm{CI})$ with adjustment a priori decided and for known confounders: 1) age 2) CCI 3) place of IHCA 4) witnessed 5) ECG-surveillance 6) first documented heart rhythm. All analyses were performed with the statistical package STATA 10.2 for Windows (STATA Corp, College Station, TX).

\section{Results}

Patient characteristics

In all, 1639 patients suffered an IHCA at Karolinska during 2007-2017 and the overall 30-day survival ratio was $29 \%$. 
Table 1 Characteristics of 1639 patients suffering an in-hospital cardiac arrest during 2007-2017 in Karolinska University Hospital

\begin{tabular}{lll}
\hline & Women Number (\%) $650(100)$ & Men Number (\%) $989(100)$ \\
\hline Age category & & \\
$18-50$ & $50(8)$ & $101(10)$ \\
$51-60$ & $84(13)$ & $117(12)$ \\
$61-70$ & $131(20)$ & $271(27)$ \\
$71-80$ & $192(30)$ & $288(29)$ \\
$\geq 81$ & $193(30)$ & $212(21)$
\end{tabular}

Charlson co-morbidity index

None 0 points

$222(34)$

Low $1-2$ points

226 (35)

346 (35)

Moderate $3-5$ points

$152(23)$

331 (33)

High 6-8 points

50 (8)

$220(22)$

National Early Warning Score 2015-2017**

240 (100)

92 (9)

Low 0-4points

97 (40)

$207(100)$

Medium 5-6 points or a single 3 point

66 (32)

High, at least 7 points

46 (19)

50 (24)

44 (18)

$31(15)$

Missing

$53(22)$

60 (29)

Place of cardiac arrest

Patient ward

$261(40)$

401 (41)

High Dependency Unit

$163(25)$

234 (24)

Intensive care unit

57 (9)

$105(11)$

Operating/procedure rooms

$11(2)$

38 (6)

Angio cath lab

$66(10)$

$19(2)$

Emergency Department

$54(8)$

ECG-surveillance

299 (46)

509 (51)

343 (53)

453 (46)

No

27 (3)

Witnessed cardiac arrest

$\begin{array}{ll}522(80) & 766(72) \\ 119(18) & 206(21) \\ 9(1) & 17(2)\end{array}$

Yes

No

First documented heart rhythm

$\begin{array}{ll}105(16) & 241(24) \\ 422(65) & 623(63) \\ 123(19) & 125(13)\end{array}$

PEA/asystole

123 (19)

125 (13)

CRP duration (minutes)

$\begin{array}{llr}\text { All, Median (IQR) } & 8(2-18) & 6(2-16) \\ \text { ROSC } & 5(2-11) & 5(2-12) \\ \text { No ROSC } & 22(14-35) & 20(12-30)\end{array}$

ROS

Yes

$490(50)$

Decision to withdraw/withhold life-sustainable treatments among those having ROSC 2015-2017
Yes
$22\left(17^{\boldsymbol{\epsilon}}\right)$

$27\left(8^{* * *}\right)$

Hypothermia/Target Temperature Management

Yes

$66\left(13^{* * *}\right)$

0.11

* $P$-values were assessed with Chi2-test regarding differences between women and men

** NEWS was only available during the years 2015-2017

$€$ Information only available for IHCA during the years 2015-2017, i.e. out of 240 women and 207 men having an IHCA, 150 (63\%) and 124 (60\%) had ROSC, respectively. Percentage 25/150 and 22/124 respectively

*** Percentage $=$ number with treatment/number with $\mathrm{ROSC}$ 
The portion of women was $40 \%(n=650)$ and their 30-days survival was $30 \%$ compared to $28 \%$ for the $989(60 \%)$ men. There was an almost equal distribution age among women and men except for that more men were aged $61-70$ years ( $27 \%$ versus $20 \%$, respectively) and more women aged at least 81 years (30 and $21 \%$, respectively, $p$-value $<0.01$, Table 1 ). No statistically significant differences were found regarding Charlson co-morbidity Index.

\section{Cardiac arrest factors}

Regarding preceding warning signs, no statistically significant difference was found regarding NEWS category among men and women, however more men had higher categories and missing information (Table 1). More men than women suffered their IHCA under ECG-surveillance (51\% versus $46 \%$, $p$-value $<0.01$, Table 1). Regarding the first documented rhythm, the portion of non-shockable were equal but more men had shockable rhythms and more women had missing information ( $\mathrm{p}$-value $<0.01$, Table 1 ). Duration of CPR attempts was equal between men and women both among those having ROSC and those not having ROSC (Table 1).

Likewise, no statistically significant differences were found regarding place of IHCA, witnessed or not or portion having ROSC.

\section{Post- ROSC factors}

No statistically significant difference was found on any post-ROSC variable, i.e. a decision to withdraw/withhold life-sustainable treatments among those having ROSC or treatment with hypothermia (2007-2015) or target temperature management (2016-2017) (Table 1).

\section{Association between sex and 30-day survival}

Men had an almost equal OR for 30-day survival compared to women (crude OR 0.93 95\% CI 0.74-1.15) that remained after adjustment for known confounders (adjusted OR 0.77 95\% CI 0.58-1.03, Table 2).

\section{Discussion}

This cohort study over 10 years illuminates an almost equal distribution of patient characteristics and cardiac arrest factors and possibly therefore an equal 30-day survival after an IHCA for men and women.

Still this study confirms previous findings of disadvantageous prerequisites such as higher age in women and more often shockable rhythm in men, but also indicates that men might have more severely deranged vital signs or not having their vital signs checked equally often as women which deserves future attention and might indicate residual confounding.

The results on age and shockable rhythm are in line with previous studies $[2,15]$, but no previously published study has investigated sex differences in CCI, NEWS and decision to withhold/withdraw treatment post-ROSC among a cardiac arrest population, and therefore comparisons to other studies are not possible. A factor affecting both the incidence to have an IHCA and the outcome of such is the admission to intensive care. Previous studies have shown that women are less likely to receive early invasive strategies such as PCI [16-18] as well as being admitted to ICU as well as received less advanced treatment while at the ICU, discussed to be a consequence of more assessments by Rapid Response Teams in men and more decisions to withhold/withdraw treatment in women [19]. However this could not be shown within our study. Interestingly, the same research group has shown that there is no difference in willingness to admit men or women to ICU but female physicians tended to be more willing to admit patients, regardless of the patient being a man or a woman, than their male counterparts [20]. Also, women tend to be placed in ordinary internal medicine unites instead of CCU's [21, 22].

Regarding the previously shown increased early survival, i.e. ROSC or admitted alive to hospital for women $[3,4]$, we found no difference in either portion having ROSC or CPR durations between men and women.

The disadvantageous prerequisites for women compared to men in studies regarding OHCA were identified as higher age, more likely to present with an OHCA in a non-public space, less likely to have a witnessed OHCA and a lower frequency of initial shockable rhythm, [2]. Solely age and initial rhythm are directly translatable in a hospital setting, which causes issues when comparing with this study.

Limitations of the study include residual confounding in variables not captured within this study such as PCI and etiology of the cardiac arrest which is important since cardiac etiologies are more common in shockable rhythms than non-shockable one. Further, factors involved in and affecting daily care not even known to clinicians and researchers are a major limitation. Further, we lack information about intake of estrogen among women, however Swedish guidelines do not recommend estrogen in women of the ages having IHCA [21].

Strengths include the inclusion of two separate different hospital-sites over ten years. Further, collection of data was based on a strict a priori determined protocol and another strength is the complete information about

Table 2 Association between sex and 30-day survival after in-hospital cardiac arrest at Karolinska University Hospital in 2007-2017

\begin{tabular}{lllll}
\hline Sex & Number of patients & 30 days survival Number (\%) & Crude OR (95\% Cl) & Adjusted OR (95\% Cl)* \\
\hline Women & 650 & $193(30)$ & 1.00 (Reference) & 1.00 (Reference) \\
Men & 989 & $278(28)$ & $0.93(0.74-1.15)$ & $0.77(0.58-1.03)$ \\
\hline
\end{tabular}

*Adjusted for age, Charlson Comorbidity Index [23], location of cardiac arrest, first documented heart rhythm, ECGsurveillance and witnessed or not 
the outcome, i.e. 30-day survival or not due to Swedish Personal Identification Numbers and registries [14].

In conclusion, this is the first study following patients with IHCA comparing men and women over 10 years. We found equal distribution of patient characteristics and cardiac arrest factors and possibly therefore an equal 30-day survival after an IHCA for men and women.

However, our study confirms previous findings of disadvantageous prerequisites such as higher age in women and more often shockable rhythm in men, but also indicates that men might have more severely deranged vital signs or not having their vital signs checked equally often as women which deserves future attention and might indicate residual confounding.

\section{Abbreviations}

CCl: Charlson co-morbidity index; CPR: Cardio-pulmonary resuscitation; ICU: Intensive care unit; IHCA: In-hospital cardiac arrest; Karolinska: Karolinska University Hospital; NEWS: National Early Warning Score; OHCA: Out-ofhospital cardiac arrest; OR, 95\% Cl: Odds Ratios with 95\% Confidence Intervals; PCI: Percutan coronary intervention; PEA: Pulseless electric activity; ROSC: Return of spontaneous circulation; SRCR: Swedish Registry for CardioPulmonary Resuscitation; VF: Ventricle fibrillation; VT: Ventricle tachycardia

\section{Acknowledgements}

The study was supported by the Karolinska Institutet.

\section{Funding}

None.

\section{Availability of data and materials}

on request from last author. No additional data exists that is suitable for publication since data are based on medical records of individuals.

\section{Authors' contributions}

All authors of this manuscript have directly participated in the planning, execution, and analyses of the study. All authors have read and approved the final version of the submitted manuscript. There are no directly related manuscripts or abstracts, published or unpublished, by any of the authors of this paper.

\begin{tabular}{|c|c|c|c|c|c|c|}
\hline Detailed author contribution & $\mathrm{AQ}$ & $C B$ & $\mathrm{JOM}$ & $\mathrm{MR}$ & KSG & TD \\
\hline Study concept and design & $x$ & $x$ & $x$ & $x$ & $x$ & $x$ \\
\hline Acquisition of data & $x$ & $x$ & $x$ & $x$ & & $x$ \\
\hline Analysis and interpretation of data & $x$ & $x$ & & & $x$ & $x$ \\
\hline Drafting of the manuscript & $x$ & & & & & $x$ \\
\hline $\begin{array}{l}\text { Critical revision of the manuscript for } \\
\text { important intellectual content }\end{array}$ & $x$ & $x$ & $x$ & $x$ & $x$ & \\
\hline Obtained funding & & & & & & $x$ \\
\hline Administrative, technical, or material support & & & & & & $x$ \\
\hline Study supervision & & & & & & $x$ \\
\hline
\end{tabular}

Competing interest

The authors declare that they have no competing interest.
Ethics approval and consent to participate

The Regional Ethical Review Board in Stockholm, Sweden approved the study, Dnr 2013/1959-31/4. Only patient that consent to participate in the registry or died due to the IHCA was included.

\section{Consent for publication}

NA

\section{Publisher's Note}

Springer Nature remains neutral with regard to jurisdictional claims in published maps and institutional affiliations.

\section{Author details}

${ }^{1}$ Function of Emergency Medicine, Karolinska University Hospital, Stockholm, Sweden. ${ }^{2}$ Department of Medicine Solna, Cardiac Unit, Center for Gender Medicine, Karolinska Institutet and University Hospital, Solna, Sweden. ${ }^{3}$ Department of Medicine Solna, Karolinska Institutet, Stockholm, Sweden.

Received: 18 October 2018 Accepted: 4 December 2018

Published online: 19 December 2018

\section{References}

1. Israelsson J, Bremer A, Herlitz J, et al. Health status and psychological distress among in-hospital cardiac arrest survivors in relation to gender. Resuscitation. 2017. https://doi.org/10.1016/j.resuscitation.2017.02.006.

2. Al-Dury N, Rawshani A, Israelsson J, et al. Characteristics and outcome among 14,933 adult cases of in-hospital cardiac arrest: a nationwide study with the emphasis on gender and age. Am J Emerg Med. 2017;35(12):183944. https://doi.org/10.1016/j.ajem.2017.06.012.

3. Bray JE, Stub D, Bernard S, et al. Exploring gender differences and the "oestrogen effect" in an Australian out-of-hospital cardiac arrest population. Resuscitation. 2013;84(7):957-63. https://doi.org/10.1016/j.resuscitation.2012. 12.004 .

4. Bougouin W, Mustafic $H$, Marijon $E$, et al. Gender and survival after sudden cardiac arrest: a systematic review and meta-analysis. Resuscitation. 2015;94: 55-60. https://doi.org/10.1016/j.resuscitation.2015.06.018.

5. Kitamura T, Iwami T, Nichol G, et al. Reduction in incidence and fatality of out-of-hospital cardiac arrest in females of the reproductive age. Eur Heart J. 2010;31(11):1365-72. https://doi.org/10.1093/eurheartj/ehq059.

6. Khan AM, Kirkpatrick JN, Yang L, et al. Age, sex, and hospital factors are associated with the duration of cardiopulmonary resuscitation in hospitalized patients who do not experience sustained return of spontaneous circulation. J Am Heart Assoc. 2014;3(6):e001044. https://doi. org/10.1161/JAHA.114.001044.

7. Hessulf F, Karlsson T, Lundgren P, et al. Factors of importance to 30-day survival after in-hospital cardiac arrest in Sweden - a population-based register study of more than 18,000 cases. Int J Cardiol. 2018;255:237-42. https://doi.org/10.1016/j.jicard.2017.12.068.

8. Stromsoe A, Svensson L, Axelsson AB, et al. Validity of reported data in the Swedish cardiac arrest register in selected parts in Sweden. Resuscitation. 2013;84(7):952-6. https://doi.org/10.1016/j.resuscitation.2012.12.026.

9. J H. Swedish CardioPulmonary register- yearly report 2014. In: Swedish; 2015.

10. Claesson A, Djarv T, Nordberg P, et al. Medical versus non medical etiology in out-of-hospital cardiac arrest-changes in outcome in relation to the revised Utstein template. Resuscitation. 2017;110:48-55. https://doi.org/10. 1016/j.resuscitation.2016.10.019.

11. Charlson M, Szatrowski TP, Peterson J, et al. Validation of a combined comorbidity index. J Clin Epidemiol. 1994:47(11):1245-51.

12. Sundararajan V, Henderson T, Perry $C$, et al. New ICD-10 version of the Charlson comorbidity index predicted in-hospital mortality. J Clin Epidemiol. 2004;57(12):1288-94. https://doi.org/10.1016/j.jclinepi.2004.03.012.

13. Roberts D, Djarv T. Preceding national early warnings scores among inhospital cardiac arrests and their impact on survival. Am J Emerg Med. 2017 https://doi.org/10.1016/j.ajem.2017.04.072.

14. Ludvigsson JF, Otterblad-Olausson P, Pettersson BU, et al. The Swedish personal identity number: possibilities and pitfalls in healthcare and medical research. Eur J Epidemiol. 2009;24(11):659-67. https://doi.org/10.1007/ s10654-009-9350-y.

15. Hessulf F, Karlsson T, Lundgren $P$, et al. Factors of importance to 30-day survival after in-hospital cardiac arrest in Sweden - a population-based 
register study of more than 18,000 cases. Int J Cardiol. 2017. https://doi.org/ 10.1016/j.jijcard.2017.12.068.

16. Bougouin W, Dumas F, Marijon E, et al. Gender differences in early invasive strategy after cardiac arrest: insights from the PROCAT registry. Resuscitation. 2017;114:7-13. https:/doi.org/10.1016/.j.resuscitation.2017.02.005.

17. Johnston N, Bornefalk-Hermansson A, Schenck-Gustafsson $\mathrm{K}$, et al. Do clinical factors explain persistent sex disparities in the use of acute reperfusion therapy in STEMI in Sweden and Canada? Eur Heart J Acute Cardiovasc Care. 2013;2(4):350-8. https://doi.org/10.1177/2048872613496940.

18. Alabas $\mathrm{OA}$, Gale $C P$, Hall $M$, et al. Sex differences in treatments, relative survival, and excess mortality following acute myocardial infarction: National Cohort Study Using the SWEDEHEART registry. J Am Heart Assoc. 2017;6(12). https://doi.org/10.1161/JAHA.117.007123.

19. Jaderling $G$, Bell $M$, Martling $C R$, et al. Limitations of medical treatment among patients attended by the rapid response team. Acta Anaesthesiol Scand. 2013;57(10):1268-74. https://doi.org/10.1111/aas.12202.

20. Larsson $E$, Zettersten $E$, Jaderling $G$, et al. The influence of gender on ICU admittance. Scandinavian journal of trauma, resuscitation and emergency medicine. 2015;23:108. https://doi.org/10.1186/s13049-015-0191-2.

21. Schenck-Gustafsson $\mathrm{K}$, Brincat M, Erel $\mathrm{CT}$, et al. EMAS position statement managing the menopause in the context of coronary heart disease. Maturitas. 2011;68(1):94-7. https://doi.org/10.1016/..maturitas.2010.10.005.

22. Herlitz J, Dellborg M, Karlsson T, et al. Treatment and outcome in acute myocardial infarction in a community in relation to gender. Int J Cardiol. 2009;135(3):315-22. https://doi.org/10.1016/j.jijcard.2008.03.065.

23. Quan H, Li B, Couris CM, et al. Updating and validating the Charlson comorbidity index and score for risk adjustment in hospital discharge abstracts using data from 6 countries. Am J Epidemiol. 2011;173(6):676-82. https://doi.org/10.1093/aje/kwq433.

Ready to submit your research? Choose BMC and benefit from:

- fast, convenient online submission

- thorough peer review by experienced researchers in your field

- rapid publication on acceptance

- support for research data, including large and complex data types

- gold Open Access which fosters wider collaboration and increased citations

- maximum visibility for your research: over $100 \mathrm{M}$ website views per year

At $\mathrm{BMC}$, research is always in progress.

Learn more biomedcentral.com/submissions 